\title{
Laser-induced film ejection at interfaces: Comparison of the dynamics of liquid and solid films
}

\author{
P. Frank $\cdot$ J. Graf $\cdot$ F. Lang $\cdot$ J. Boneberg $\cdot$ P. Leiderer
}

\begin{abstract}
The ejection dynamics of nanometer-thin fluid isopropanol and solid $\mathrm{CO}_{2}$ films are investigated. The films are deposited on a silicon substrate, which is rapidly heated by a nanosecond laser pulse (Nd:YAG, $532 \mathrm{~nm}$ ). A small fraction of material at the interface evaporates and the film on top is ejected as an intact layer. The kinetic energies of the two different films with thicknesses between $100 \mathrm{~nm}$ and $1 \mu \mathrm{m}$ give an insight into the dynamics of a flying lamella.
\end{abstract}

\section{Introduction}

The dynamics of the film ablation are of substantial interest for a wide variety of applications, such as laser cleaning of nanoparticles from sensitive surfaces [1,2], laser desorption mass spectroscopy [3], or laser surgery [4]. The existing investigations deal mostly with the phase transition at interfaces [5-8], nucleation on the nanoscale, and massive ablation of materials [4]. Studies on the mechanisms of steam laser cleaning and matrix laser cleaning [9] have shown that films ablated by that means can be observed during some hundred nanoseconds $[10,11]$. The investigation of the dynamics of the film ejection at interfaces is a new component in the overall picture for ablation processes involving liquid or solid films.

The basic idea of the experiments is the examination of the ablation dynamics of a transparent microlamella by means of the desorption of a thin film condensed on a flat substrate with a short $(10 \mathrm{~ns})$ and intense laser pulse $[6,11]$. The first few monolayers of the film in contact with the substrate overheat and evaporate rapidly. As the vapor expands,

P. Frank (®) · J. Graf · F. Lang · J. Boneberg · P. Leiderer University of Konstanz, Fach 676, 78457 Konstanz, Germany e-mail: Pascal.Frank@uni-konstanz.de the remaining film is accelerated away from the surface (see Fig. 1). Monitoring the film velocities with nanosecond time-resolved reflectometry gives an insight into the overall energy balance of the thin-film ablation.

\section{Experimental setup}

In the following, two experimental setups are presented. They do not differ substantially except for the used adsorbed medium. The substrate is in both cases a silicon wafer, which is cleaned in advance by standard methods to provide a smooth and uncontaminated surface. More detailed descriptions can be found in Refs. [6] and [9].

To produce a liquid film, isopropanol $\left(\mathrm{C}_{3} \mathrm{H}_{7} \mathrm{OH}\right)$ vapor at slightly elevated temperature (approx. $30^{\circ} \mathrm{C}$ ) is blown over the substrate. As isopropanol wets the silicon completely it condenses homogeneously. The thickness of the layer is determined with the reflectometry by comparing the relative intensity to values calculated using Fresnel's formula. The accuracy is better than $10 \mathrm{~nm}$. An example for the signal of a growing layer is shown in Fig. 2 .

To produce a solid film, $\mathrm{CO}_{2}$ vapor is condensed onto a silicon substrate in a vacuum chamber at $1 \mathrm{mbar}$ cooled down to $77 \mathrm{~K}$. The films on the substrate are quenchcondensed polycristalline and microporous films [12]. The thickness measurements are done the same way as for the liquid films. By comparing the absolute values of the amplitude of the reflectrometry measurements to the MaxwellGarnett theory [13] a filling factor of over $70 \%$ is deduced. The size of the pores is estimated to be below $50 \mathrm{~nm}$ due to the fact that no scattered light is observed.

An intense laser pulse (Nd:YAG, $\lambda=532 \mathrm{~nm}, \mathrm{FWHM}=$ $10 \mathrm{~ns})$ then hits the prepared site. Since the adsorbed layer is transparent, the laser pulse is absorbed in the silicon and 
Fig. 1 Evaluation of the vapor cavity. The intense light pulse heats the substrate. A small portion of the adsorbed medium evaporates and accelerates the remaining layer, which stays at lower temperatures

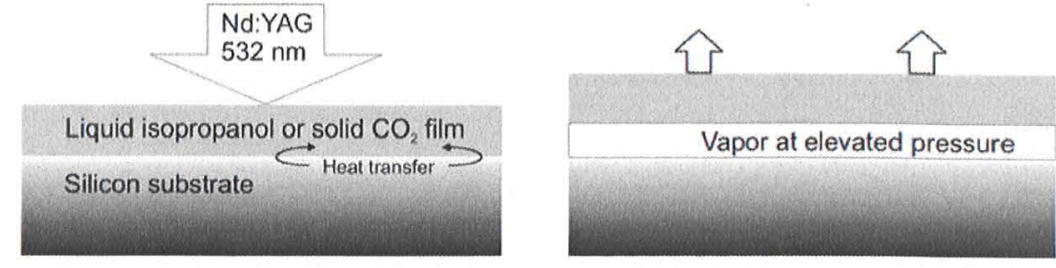

Fig. 2 Reflectometry signal of a growing isopropanol layer on silicon. The laser shot takes place at $0 \mathrm{~s}$

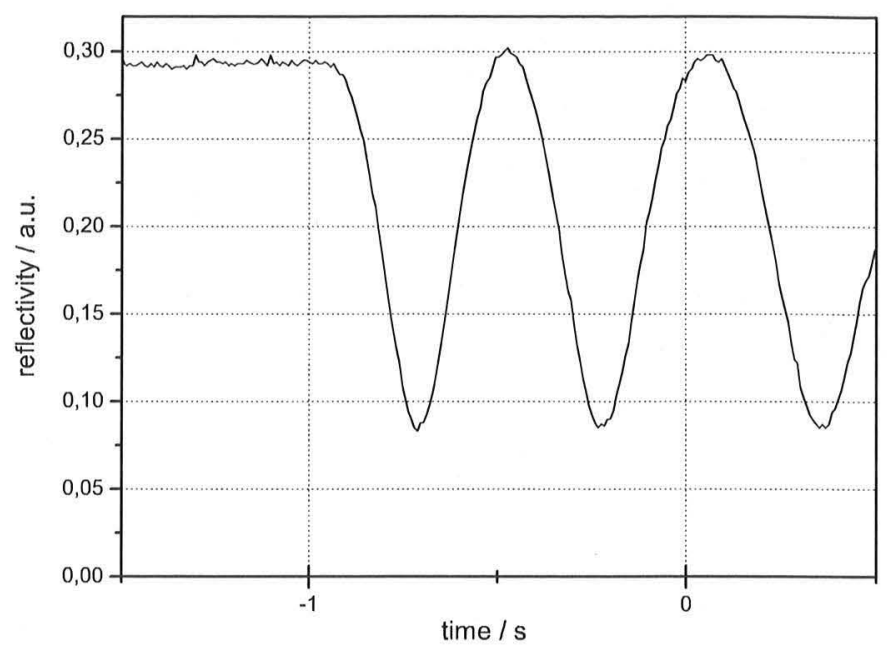

the heat is subsequently transferred to the first few monolayers of the film medium. The diffusion length in a silicon is around $800 \mathrm{~nm}$ on a timescale of $10 \mathrm{~ns}$, in isopropanol and solid $\mathrm{CO}_{2}$ the value is three orders of magnitude smaller. As the heating rates are in the order of $10^{10}, \mathrm{~K} / \mathrm{s}$, the medium is likely to overheat and the subsequent phase transition generates a vapor cavity at high pressure, which gives rise to the ejection of the film.

A fast reflectometer with temporal resolution below $1 \mathrm{~ns}$ monitors the motion of the film due to the interference of the reflections at the substrate surface and the two interfaces of the desorbed film as outlined in Fig. 3. The interferometer has high temporal $(<1 \mathrm{~ns})$ and spatial accuracy $(<10 \mathrm{~nm})$ regarding the movement in $z$-direction. Typical signals of moving films are shown in Figs. 4 and 6.

\section{Results}

\subsection{Fluid films}

The signal of the flying liquid film in Fig. 4 shows four different stages:

Immediately after the laser pulse, the reflectivity changes significantly and this change is periodical with an increasing period (A). At approximately $180 \mathrm{~ns}$, the signal is nearly constant (B). Afterwards the oscillations in intensity reappear but the period decreases mirror-image-like to the first

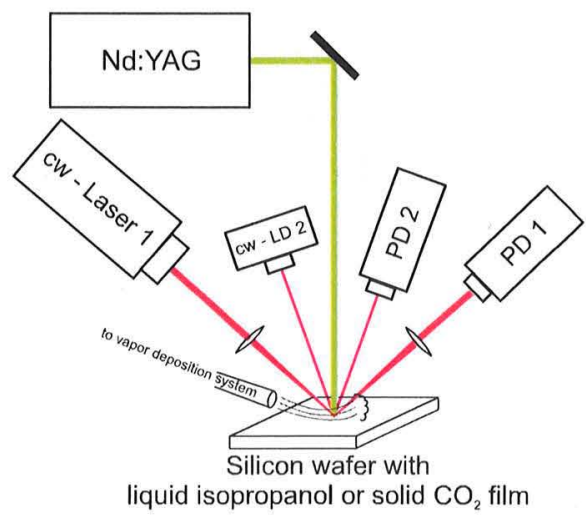

Fig. 3 Sketch of the experimental setup. The Nd:YAG laser pulse $(\lambda=532 \mathrm{~nm}, \mathrm{FWHM}=10 \mathrm{~ns})$ heats the substrate. The monitoring system is made up of two reflectometers, the first consisting of Laser 1 -either a laser diode $(\lambda=655 \mathrm{~nm})$ or an $\mathrm{Ar}^{+}$laser $(\lambda=488 \mathrm{~nm})$ - and the fast photodiode 1 with sub-ns resolution for the fast dynamics. The second system for the film thickness measurements consists of a small $\mathrm{CW}$ laser diode and a relatively slow photodiode (LD 2 and PD 2). The vacuum chamber for $\mathrm{CO}_{2}$ films is omitted

stage (C). After approximately $350 \mathrm{~ns}$, the signal drops back to the initial value (D). The signal does not show any significant features on a longer timescale. The change of the reflected intensity depends on the particular layer thickness. In the presented case, it rises. The signal originates from the expansion and collapse of the vapor cavity between the substrate and the moving film. Calculations for the system 


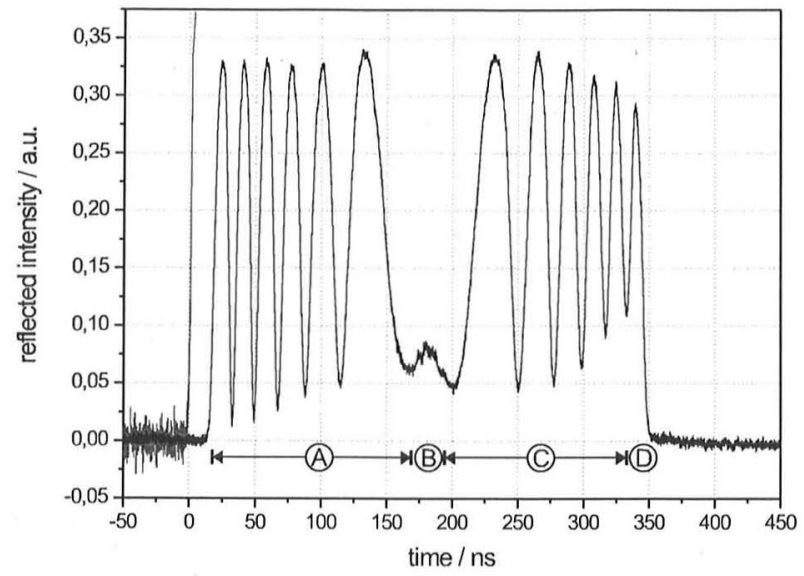

Fig. 4 Reflected signal of a $620-n m$ thick isopropanol film after the laser pulse that is released at $0 \mathrm{~ns}$, with approximately $30 \mathrm{~mJ} /$ pulse. The signal shows the desorption and the return of the film

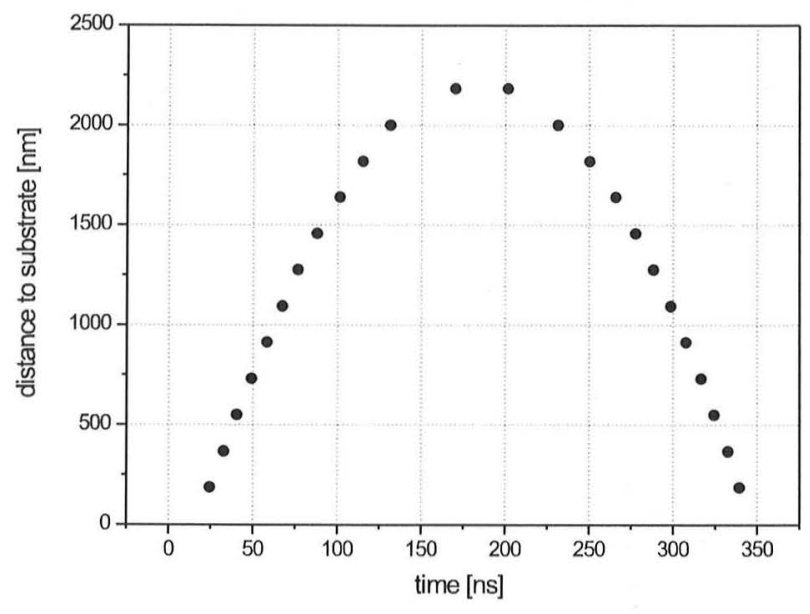

Fig. 5 Calculated distance of the film in Fig. 4 to the surface of the substrate. The film trajectory is nearly parabolic. The data from the first $50 \mathrm{~ns}$ are used to determine the starting velocity of the film consisting of an isopropanol layer of $620 \mathrm{~nm}$ in thickness, a varying thickness of the vapor cavity and the substrate using Fresnel's formula allow to allocate the peaks in the measured signal (Fig. 4), giving a nearly parabolic trajectory as shown in Fig. 5. The initial slope allows to determine the starting velocity of the films, from which the information about the energetics of the ejection process can be obtained.

\subsection{Solid films}

In Fig. 6, the signal showing the change of reflectivity upon a laser shot is depicted for the case of a solid $\mathrm{CO}_{2}$ film. The reflectivity rises after the onset of the laser pulse and oscillates with nearly constant period.

The starting velocities of the liquid and solid films (see Fig. 7) for film thicknesses between 100 and $1000 \mathrm{~nm}$ and 100 to $500 \mathrm{~nm}$, respectively, show a similar behavior: they are decreasing for thicker layers. A more detailed comparison, however, reveals differences which are discussed later in the text.

\section{Discussion}

As already pointed out, the local explosive evaporation results in a small gas cavity between the film and the substrate with a pressure in the MPa regime [6, 11]. The subsequent expansion of this vapor cavity causes the acceleration of the film. The expansion of this cavity leads to an oscillating interference between the reflected light of the surface of the substrate and the two surfaces of the film. In the liquid case, the pressure in the cavity diminishes during the flight, the film slows down and eventually the direction of motion is reversed and the film returns to the substrate. This behavior is due to the ambient pressure pushing from above. For solid films, on the other hand, the experiments are carried out under vacuum conditions, hence there is no ambient gas
Fig. 6 Signal of a desorbing solid $\mathrm{CO}_{2}$ film. Due to the low ambient pressure, the film does not return, but flies with constant velocity

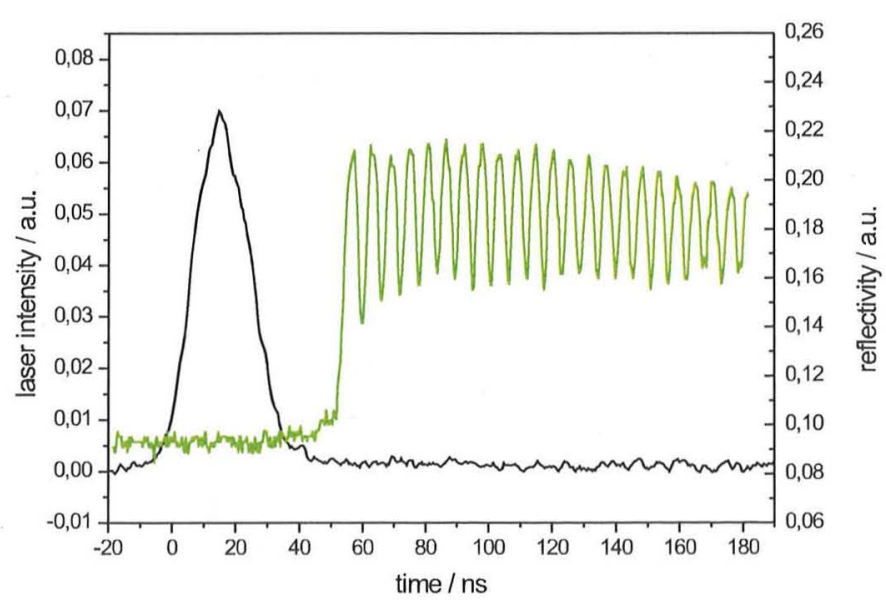




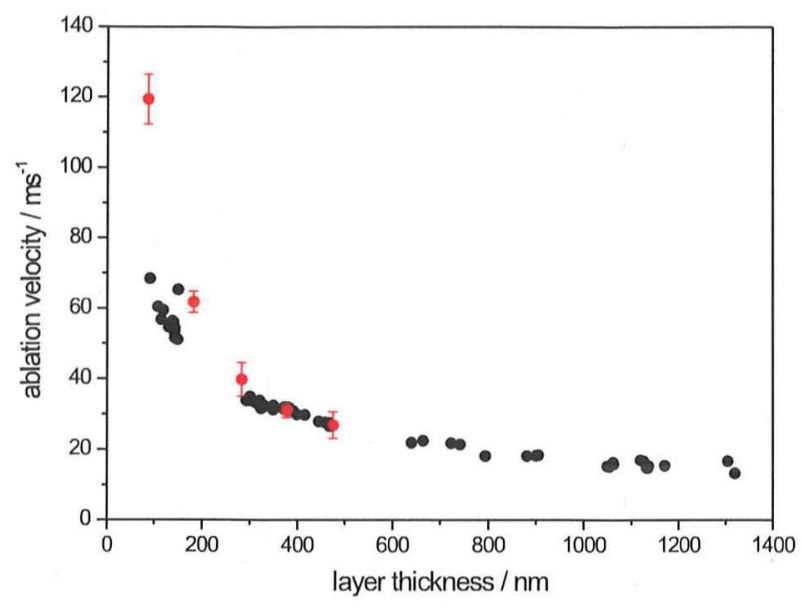

Fig. 7 Velocity of the detached films right after desorption of the substrate depending of the initial thickness of the film. Black: fluid films - isopropanol on silicon; red: solid films $-\mathrm{CO}_{2}$ on silicon

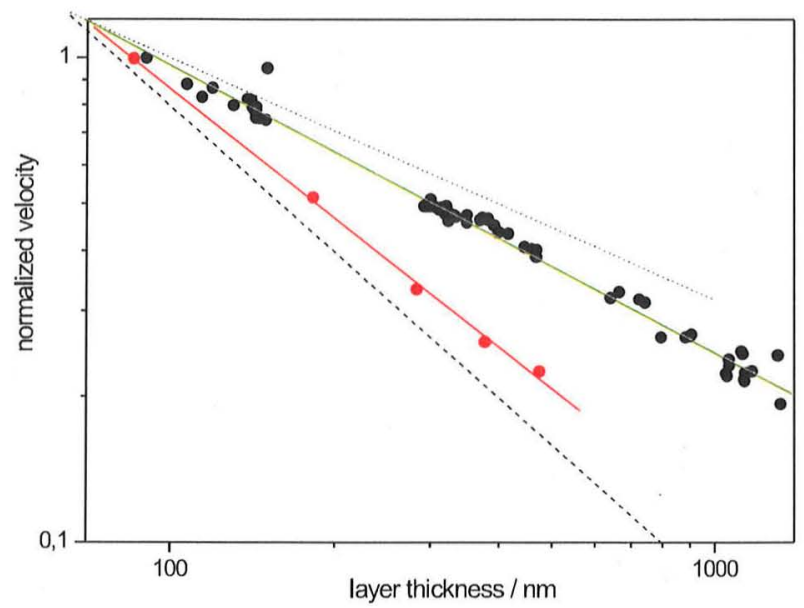

Fig. 8 Normalized velocities of the films presented in Fig. 7 on a double logarithmic scale, which reveals a clear difference between solid (red) and fluid (black) films. The fitting function is of the form: $v \propto d^{m}$. Dashed line: $m=-1$. Dotted line: $m=-0.5$

pressure from above, and the film moves away from the substrate at constant velocity. In order to compare the ejection of solid and liquid films in more details, we plot the data of Fig. 7 on a double logarithmic scale (Fig. 8), with values normalized to the velocities of the fastest, i.e., thinnest films. The behavior of both films is well presented by a power law: the velocity $v$ is proportional to the thickness of the layer $d$ to the power $m$, which corresponds to the slope of the fitting lines. The values are $m=-0.9$ for the solid films and $m=-0.6$ for liquid films.

The energy deposited in the substrate with the laser pulse is kept constant for each of the two cases within the experimental limits. The potential energy $E_{\text {pressure }}$ of the gas layer directly after its formation on a subnanosecond timescale [6] determines the maximal kinetic energy $E_{\text {kin }}$ of the films after the acceleration. The different dissipation mechanisms during this process are summarized to the losses, some of which are dependent on the layer thickness $d, E_{\text {loss }}(d)$, and some of which are not, $E_{\text {loss }}$ (other), i.e.,

$E_{\text {pressure }}=E_{\mathrm{kin}}(d)+E_{\mathrm{loss}}(d)+E_{\mathrm{loss}}($ other $)$

The loss of the energy during the acceleration of the film occurs through various channels. These can be vibrational or acoustic excitations in the film or in the bulk of the underlying substrate, and a reduction of the pressure at the edges of the ablated region which is imaginable even if the observed parts are in the middle of a much bigger irradiated spot. The different dependencies of the starting velocity $v$ (and hence of $E_{\text {kin }}$ ) on the film thickness reveal different dissipation mechanisms in solid and liquid films. If the dissipation is due to effects, which are layer-thickness independent, the energy of the film should not depend on the layer thickness. Thus:

$E=\frac{1}{2} \cdot A d \rho \cdot v^{2}+E_{\text {loss }}=$ constant $\Rightarrow v \propto \frac{1}{\sqrt{d}}$

The liquid films follow this behavior quite closely. The dissipation during the transition of the kinetic energy of the vapor molecules to the isopropanol film is only weakly dependent on the thickness of the layer.

In the case of the solid films, a different scenario occurs. In spite of the similarities in the ablation kinetics, the dissipation strongly depends on the layer thickness. It is conceivable that the solid, microporous $\mathrm{CO}_{2}$ is a strong absorber of acoustic excitations, which occur at the interface and might carry more energy when the film thickness increases. However, a detailed model is so far missing.

\section{Conclusion}

We presented studies of the ablation dynamics of liquid and solid films, which are ejected from a rapidly heated surface. The films remain intact during the time of flight. The observation of the flight trajectory by means of reflectometry gives the access to the velocity and thus the kinetic energy directly after the acceleration by the expanding gas cushion formed at the laser heated substrate. Despite all the experimental similarities the energetical comparison of the two systems reveals a significant difference between solid and liquid films concerning the dependence of energy dissipation on the layer thickness. The energy dissipation in liquid films is nearly independent of the thickness, whereas the dissipation in solid films strongly depend on it. The origin of this difference might be due to the different film morphologies resulting in a different acoustic damping but further investigations are necessary to clarify this point. 
Acknowledgements Financial support by the Center of Applied Photonics (CAP) at the University of Konstanz and the Deutsche Forschungsgemeinschaft (DFG) project number LE 315/22-1 is gratefully acknowledged.

\section{References}

1. A.C. Engelsberg, Dissertation, Rensselaer Polytechnique Institute, 1988

2. W. Zapka, W. Ziemlich, A. Tam, Appl. Phys. Lett. 58, 20 (1991)

3. S. Berkenkamp, F. Kirpekar, F. Hillenkamp, Science 281, 260-262 (1998)
4. A. Vogel, V. Venugopalan, Chem. Rev, 103, 577-644 (2003)

5. S. Kudyrashov, S. Allen, J. Appl. Phys. 95, 5820 (2004)

6. F. Lang, P. Leiderer, New J. Phys. 8, 14 (2006)

7. A. Samokhin, Quantum Electron. 36, 648-686 (2006)

8. Y. Dou, L. Zhigilei, N. Winograd, B. Garrison, J. Phys. Chem. A 105, 2748-2755 (2001)

9. J. Graf, B. Lukyanchuk, M. Mosbacher, M.H. Hong, C.T. Chong, J. Boneberg, P. Leiderer, Appl. Phys. A 88, 2 (2007)

10. F. Lang, S. Georgiou, P. Leiderer, Appl. Phys. Lett. 85, 14 (2004)

11. J. Graf, PhD thesis, University of Konstanz, 2007

12. K. Müller, Surf. Sci. 184(1-2), L375-L382 (1987)

13. J. Maxwell-Garnett, Philos. Trans. R. Soc. Lond. 203, 385 (1904) 